\title{
A Computer Model for the Simulation of Nonspherical Particle Dynamics in the Human Respiratory Tract
}

\author{
Robert Sturm \\ Brunnleitenweg 41, A-5061 Elsbethen, Salzburg, Austria \\ Correspondence should be addressed to Robert Sturm, sturm_rob@hotmail.com
}

Received 29 April 2011; Accepted 21 October 2011

Academic Editor: Anil Chourasia

Copyright () 2012 Robert Sturm. This is an open access article distributed under the Creative Commons Attribution License, which permits unrestricted use, distribution, and reproduction in any medium, provided the original work is properly cited.

In the study presented here deposition of spheres and nonspherical particles with various aspect ratios (0.01-100) in the human respiratory tract was theoretically modeled. Shape of the nonspherical particles was considered by the application of the latest aerodynamic diameter concepts. Particle deposition was predicted by using a stochastic model of the lung geometry and simulating particle transport trajectories according to the random-walk algorithm. Concerning fibers total deposition is significantly enhanced with respect to that of spheres for $\mu \mathrm{m}$-sized particles, whereby at normal breathing conditions peripheral lung compartments serve as primary deposition targets. In the case of oblate disks, total deposition becomes mostly remarkable for submicron particles, with the bronchioli and alveoli being targeted to a high extent. Enhancement of the aerodynamic diameter and/or flow rate generally causes a displacement of deposition maxima from peripheral to more proximal lung regions. From these findings, it can be concluded that these particle classes may represent tremendous occupational hazards, especially if they are attached with radioactive elements or heavy metals.

\section{Introduction}

The shape of aerosol particles may be regarded as a determinant which affects well-known phenomena such as dry deposition, cloud scavenging, and deposition in the human respiratory tract. The majority of aerosol particles occurring in the atmosphere deviates significantly from ideal spherical shape. This category of nonspherical particles among other includes fibers, disk- or platelet-shaped particulate structures, and various kinds of agglomerates. Fibers are defined as elongated particles with an aspect ratio, that is, a ratio of the length to the diameter, greater than three [1] (Figure 1). In the past, fibrous particles were classified as notorious occupational hazards, and especially the exposure to airborne asbestos fibers was found to increase the incidence of lung cancer [2]. Therefore, enhanced scientific interest was concentrated on this particle class, and important findings regarding the extrathoracic and tracheobronchial deposition behaviour of particles with various aspect ratios were published [3-5]. In 1989 the use of asbestos fibers in the building industry was forbidden by the US Environmental Protection
Agency, leading to the introduction of substitutes with similar properties, from which man-made vitreous fibers (MMVFs) crystallised out as most appropriate [6]. However, it could be detected by experiments with laboratory animals that MMVFs have similar effects on the lung as asbestos $[7,8]$. This circumstance will preserve the high medical interest in the behaviour of fibrous particles also in future. Oblate disks represent the preferential particle shapes of dust aerosols being generated by mechanical processes such as friction, grinding, burnishing, and so forth. In contrast to fibers, their aspect ratio is much smaller than 1 (Figure 1). Studies regarding the deposition behaviour of these particles are still very scarce although the exact knowledge of dust deposition in the lung would mean a tremendous progress in aerosol sciences and lung medicine. Agglomerated particles or aggregates are usually found in many fields, whereby most of them are formed by combustion processes. High importance concerning both atmospherical and health effects has to be attributed to diesel soots which are currently produced in large amounts by all kinds of diesel engines $[9,10]$. The morphology of these particles is defined by 


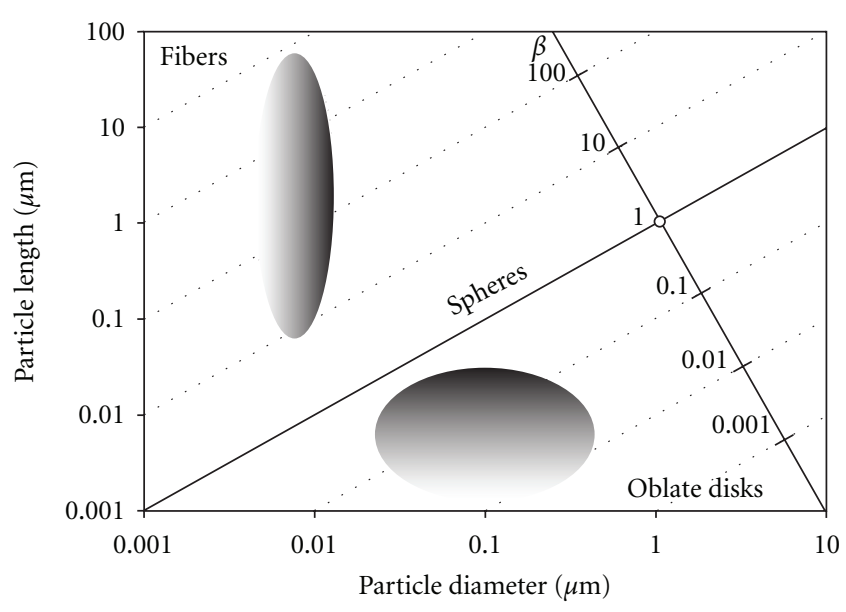

FIGURE 1: Diagram with particle length plotted against cylindrical particle diameter. While for fibers with cylindric or rod-like shapes aspect ratio $\beta$ takes values $>1$ (by convention even values $>3$ ), for oblate disks $\beta$ is characterised by values $<1$.

the so-called fractional dimension, with the help of which individual agglomerates and agglomerate populations can be characterized [11].

Theoretical approaches to the deposition of nonspherical particles in the human respiratory tract are mainly based on the concept of the aerodynamic diameter which represents the diameter of a unit-density sphere with identical settling velocity as the particle of interest. For $\mu \mathrm{m}$-sized and submicron aerosol particles, the effect of shape on particle kinetics is commonly expressed by the dynamic shape factor taking values greater than 1 in the case of nonsphericity [12]. In the past, theoretical determination of this factor succeeded in several ways, for example, by the definition of individual particle mobilities parallel and perpendicular to the airflow [13], by the calculation of fractal dimensions [11], and by the application of the so-called Geldard method [14]. Computer models solely working on the basis of the aerodynamic diameter could demonstrate that for sitting breathing conditions (tidal volume: $750 \mathrm{~mL}$, breathing frequency: $12 \mathrm{~min}^{-1}$ ) alveolar deposition of fibers with aspect ratios smaller than ten is similar to that of spheres but also remains appreciable for longer fibers [2, 13]. Comparable results for the deposition of oblate disks and agglomerates are not available at the moment. An alternative mathematical approach described the behaviour of single nonspherical particles in the flow field of airway bifurcations by the application of numerical concepts such as computational fluid dynamics (CFD). As a main result of these theoretical models, particle deposition was represented as a function of the Stokes number $(S t)$ which itself is directly proportional to particle size, particle density, and flow velocity. Computer simulations which founded upon CFD and were carried out during the past decade clearly demonstrated that bronchial deposition of fibers among other depends upon their orientation relative to the airflow [15-17]. Therefore, particles oriented perpendicular to the flow vector are characterised by a significantly increased deposition in the most proximal airway bifurcations due to the additional occurrence of interception as an essential deposition mechanism. Fibers oriented parallel to the flow vector are deposited in the bronchi to a lower extent but are preferentially transported to distal lung regions, where they may be accumulated in the alveoli. Similar CFD simulations dealing with the transport and deposition of oblate disks or agglomerates in the tracheobronchial tree are missing at the moment but might represent a remarkable research field in aerosol science.

The objectives of the study presented here are twofold. In a preceding theoretical part, the mathematical background necessary for the simulation of nonspherical particle deposition in the human respiratory tract is introduced. Main interest is focused on the computation of appropriate dynamic shape factors and resulting aerodynamic diameters of fibers and disks with different aspect ratios. In a modelling part following the theoretical remarks, the aerodynamic diameter concept is applied to a stochastic particle transport and deposition model [18] which was subject to numerous validation processes in the past and, therefore, may be evaluated as highly reliable in its category. Deposition scenarios of fibers, oblate disks, and spheres with similar dimensions are compared, and the relevance of possible differences concerning the individual deposition patterns will be discussed in detail.

\section{Materials and Methods}

As already outlined in the preceding section, the simulation of nonspherical particle deposition in the lung, which is in the centre of interest in this work, is based on the concept of aerodynamic diameters. In general, the aerodynamic diameter, $d_{\mathrm{ae}}$, of a particle with arbitrary shape and/or density is given by the formula

$$
d_{\mathrm{ae}}=d_{\mathrm{ev}} \cdot \sqrt{\frac{1}{\chi} \cdot \frac{\rho_{p}}{\rho_{0}} \cdot \frac{C_{c}\left(d_{\mathrm{ev}}\right)}{C_{c}\left(d_{\mathrm{ae}}\right)}},
$$

where $d_{\mathrm{ev}}$ denotes the so-called equivalent volume diameter, whereas $\chi, \rho_{p}, \rho_{0}, C_{c}\left(d_{\mathrm{ev}}\right)$, and $C_{c}\left(d_{\mathrm{ae}}\right)$, respectively, represent the dynamic shape factor, the density $\left(\mathrm{g} \mathrm{cm}^{-3}\right)$ of the particle, unit density $\left(=1 \mathrm{~g} \mathrm{~cm}^{-3}\right)$, the Cunningham slip correction factor for a particle with diameter $d_{\mathrm{ev}}$ as well as the correction factor for a particle with diameter $d_{\mathrm{ae}}$. The equivalent volume diameter is the diameter of a sphere with exactly the same volume as the particle of interest. Mathematically, $d_{\mathrm{ev}}$ can be defined as follows:

$$
d_{\mathrm{ev}}=\sqrt[3]{\frac{6}{\pi} \cdot V_{p}}
$$

In (2), $V_{p}$ denotes the volume of the nonspherical particle which in the case of cylindrical fibers and disks amounts to $\left(d_{p} / 2\right)^{2} \cdot\left(\pi \cdot \beta \cdot d_{p}\right)$, with $d_{p}$ and $\beta$ representing the cylindrical diameter and the aspect ratio. The dynamic shape factor, $\chi$, of an irregularly shaped and randomly orientated particle being 
TABLE 1: Dynamic shape factors of nonspherical particles, using the definition of the coefficients outlined in (4).

\begin{tabular}{lcccc}
\hline \multirow{2}{*}{ Coefficients } & \multicolumn{2}{c}{ Fibers } & \multicolumn{2}{c}{ Oblate disks } \\
& $\chi_{/ /}$ & $\chi_{\perp}$ & $\chi_{/ /}$ & $\chi_{\perp}$ \\
\hline$a_{1}$ & 8 & 8 & 4 & 4 \\
$a_{2}$ & 3 & 1 & 3 & 1 \\
$a_{3}$ & $\beta^{2}-1$ & $\beta^{2}-1$ & $1-\beta^{2}$ & $1-\beta^{2}$ \\
$a_{4}$ & $\beta+\left(\beta^{2}-1\right)^{0,5}$ & $\beta+\left(\beta^{2}-1\right)^{0,5}$ & $\beta$ & $\beta$ \\
$a_{5}$ & $\beta$ & $-\beta$ & $\beta$ & $-\beta$ \\
$F$ & $\ln$ & $\ln$ & Arccos & Arccos \\
\hline
\end{tabular}

transported in the flow field of a bronchial airway is given by the common formula

$$
\frac{1}{\chi}=\frac{1}{3 \chi_{/ /}}+\frac{2}{3 \chi_{\perp}}
$$

where $\chi_{/ /}$and $\chi_{\perp}$ are the specific dynamic shape factors of the particle oriented and moving parallel and perpendicular to the flow vector [1]. In general terms, the dynamic shape factor of a fiber (prolate shape) or disk (oblate shape) can be defined by the following approximation [19]:

$$
\chi=\frac{\left(a_{1} / 3\right) \cdot\left(\beta^{2}-1\right) \cdot \beta^{-1 / 3}}{\left(\left(2 \beta^{2}-a_{2}\right) / \sqrt{a_{3}}\right) \cdot F\left(a_{4}\right)+a_{5}} .
$$

In the formula noted above, $a_{1}-a_{5}$ represent specific numerical coefficients, whereas $F$ is a place holder for a mathematical function which is different for fibers and disks (Table 1). Whilst for fibers the approximation is valid for values of $\beta$ greater 1 , for platelets $\beta$ has to be smaller than 1. For $\beta=1$, particle shape is commonly assumed to be spherical with $\chi=1$. The Cunningham slip correction factor $C_{c}$ of (1) can be expressed by the general formula

$$
C_{c}=1+\frac{\lambda}{d_{\mathrm{ev}}} \cdot\left[2.514+0.800 \cdot \exp \left(-0.55 \cdot \frac{d_{\mathrm{ev}}}{\lambda}\right)\right] .
$$

In (5), $\lambda$ describes the mean free path of air molecules, amounting to $0.066 \mu \mathrm{m}$ at standard conditions $\left(20^{\circ} \mathrm{C}\right.$ and $1.013 \mathrm{~atm})$. To fulfil the temperature and pressure conditions in the tracheobronchial tree of the human lung, $\lambda$ has to be recalculated according to the following equation:

$$
\lambda=\frac{R_{g} \cdot T}{\sqrt{2} \cdot \pi \cdot d_{\text {air }}^{2} \cdot N_{A} \cdot P} .
$$

In the formula introduced above, $R_{g}$ is the gas constant $\left(8.344 \mathrm{~Pa} \mathrm{~m}^{3} / \mathrm{molK}\right), d_{\text {air }}$ the mean diameter of the air molecules, $N_{A}$ the Avogadro number $\left(6.022 \cdot 10^{23}\right), T$ the air temperature and $P$ the related pressure. While air temperature, in the lung takes an average value of $36^{\circ} \mathrm{C}$, pressure in single airway tubes is calculated according to the Hagen-Poiseuille law

$$
\frac{V}{\Delta t}=\frac{\pi \cdot \Delta P \cdot R^{4}}{8 \cdot \eta \cdot L} .
$$

The radius and length of a selected airway are represented by the variables $R$ and $L$, whereas $\eta$ denotes the dynamic viscosity of the fluid passing through the tube system. For air it amounts to $1.8 \times 10^{-4} \mathrm{~Pa} \cdot \mathrm{s}$ or $0.18 \mathrm{cP}$ (centipoise). $\Delta t$, which can be alternatively formulated as $t_{i}-t_{i-1}$, describes the difference of time for a given air volume to pass the airway tube $i-1$ and the following tube $i$. The same explanation can be applied to the pressure difference $\Delta P$ that can be written as $P_{i}-P_{i-1}$.

The stochastic deposition model used in this context was introduced and described in detail by Koblinger and Hofmann [18], so that only the most salient features of the approach will be subject to a brief elucidation. In general, the model is based on a random walk algorithm, where particle trajectories follow a randomly selected path through the tracheobronchial and acinar regions. The generation of a larger number (e.g., 10,000) of such particle trajectories, which is necessary for statistical reasons, was realized by the Monte Carlo simulation technique. To obtain realistic deposition scenarios, morphometric data of the tracheobronchial tree [20] as well as data of the acinar morphology [21] were implemented into the stochastic model. In order to utilize these huge amounts of published morphometric data for modelling, they were subject to extensive statistical analyses, thereby, deriving reliable probability density functions for the distributions of diameters, lengths, branching, and gravity angles $[18,20]$. For the simulation of the random path of an inhaled particle through the stochastic airway system, at each branching site linear generation-specific airway dimensions are selected from probability density functions by a pseudorandom number generator, considering also potential correlations between the morphometric parameters themselves.

In the stochastic deposition model, three main particle deposition forces, namely, inertial impaction, gravitational settling, and Brownian diffusion are distinguished. Corrected diameters for anisometric particles introduced in (1) are commonly applied to all deposition forces. For each airway tube, an individual deposition probability is computed which in the case of inertial impaction results from the formula [22]

$$
p_{I}=1-\frac{2}{\pi} \cdot \cos ^{-1}(\theta \cdot S t)+\frac{1}{\pi} \cdot \sin \left[2 \cdot \cos ^{-1}(\theta \cdot S t)\right] \text {, }
$$

where $\theta$ is the half branching angle of two daughter airways within an airway bifurcation, and $S t$ is the Stokes number. Equation (8) is only valid for $S t<1$, while for $S t \geq 1 p_{I}$ commonly amounts to 1 . Deposition probability due to gravitational settling is given by

$$
p_{S}=1-\exp \left(\frac{4 \cdot g \cdot C_{c} \cdot \rho_{p} \cdot d_{\mathrm{ae}} \cdot L \cdot \cos \Phi}{18 \cdot \pi \cdot \eta \cdot R \cdot v}\right)
$$

with $g, d_{\mathrm{ae}}, \Phi$, and $v$, respectively, representing the gravitational constant $\left(9.87 \mathrm{~kg} \mathrm{~m} \mathrm{~s}^{-2}\right)$, the aerodynamic diameter of the particle of interest, the angle of a given airway tube axis relative to the direction of gravity, and the flow velocity in 
the airway tube. Deposition probability caused by Brownian diffusion is defined by the formula

$$
p_{D}=1-\sum_{i=1}^{3} a_{i} \cdot \exp \left(-b_{i} \cdot x\right)-a_{4} \cdot \exp \left(-b_{4} \cdot x^{2 / 3}\right)
$$

In (10) $a_{i}$ and $b_{i}$ are empirical coefficients [22], while $x$ is defined as follows:

$$
x=\frac{L \cdot D}{2 \cdot R^{2} \cdot v} .
$$

$D$ denotes the diffusion coefficient of the particle with aerodynamic diameter. It is calculated for the fluid and temperature of interest according to the well-known Einstein equation. Assuming a total number of 100,000 particles, whose trajectories are simulated by the model, the amount of particles deposited in the bronchial airway $j$ is obtained according to the following considerations:

$$
N_{j}=\left[100,000 \cdot \prod_{i=1}^{j-1}\left(1-p_{i}\right)\right] \cdot p_{j} .
$$

In the formula noted above, $p_{i}$ and $p_{j}$ include the deposition probabilities for inertial impaction, gravitational settling, and Brownian diffusion which were introduced in (8)-(10).

\section{Results}

3.1. Normalized Particle Mobilities and Calculated Diameters. As demonstrated by (1)-(4), the aerodynamic diameter of a nonspherical particle mainly depends upon the equivalent volume diameter of that particle. In addition $d_{\mathrm{ae}}$ is also affected to a certain extent by the dynamic shape factor $\chi$ which is derived from the components $\chi_{/ /}$and $\chi_{\perp}$ according to (3). Since $\chi$ does not depend upon particle size, computation of the normalized mobilities $b_{/ /}$and $b_{\perp}$ was carried out, thereby using the simple relationship $b=\chi \cdot C_{c}$ (Figure 2(a)). Normalized particle mobilities of oblate disks with an aspect ratio of 0.1 are characterized by an exponential decrease with increasing cylindrical diameter $d_{p}$ that is, small particles $\left(d_{p}<0.1 \mu \mathrm{m}\right)$ exhibit significant mobilities, whereas for large particles $\left(d_{p}>1 \mu \mathrm{m}\right) b_{/ /}$and $b_{\perp}$ may be evaluated as nearly negligible. Within the $d_{p}$ range from 0.001 to $0.1 \mu \mathrm{m}, b_{\perp}$ commonly exceeds $b_{/ /}$by a factor of 1.1 to 1.2 . A similar behaviour of the normalized particle mobilities may be recognized for fibers with an aspect ratio of 10 although computed values of $b_{/ /}$and $b_{\perp}$ are significantly lower with respect to oblate disks (Figure $2(\mathrm{a})$ ).

The calculated diameters $d_{\mathrm{ev}}$ and $d_{\mathrm{ae}}$ are summarized in Figure 2(b) and Table 2, whereby unit-density particles $\left(\rho_{p}=\rho_{0}\right)$ were assumed for the computations. As an important fact, $d_{\mathrm{ev}}$ exceeds $d_{\mathrm{ae}}$ for fibrous as well as disklike particles due to $\chi$ taking values $>1$ within both particle categories. Based upon the respective geometry, calculated diameters of oblate disks are commonly lower than related

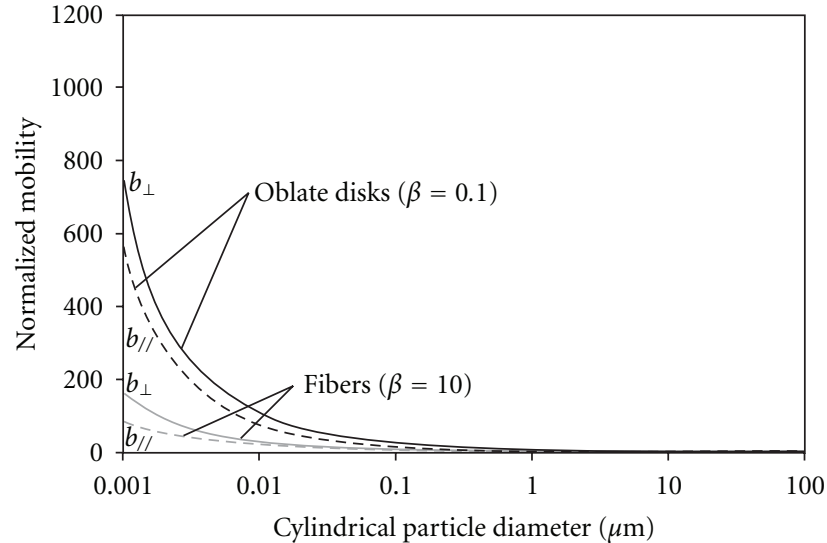

(a)

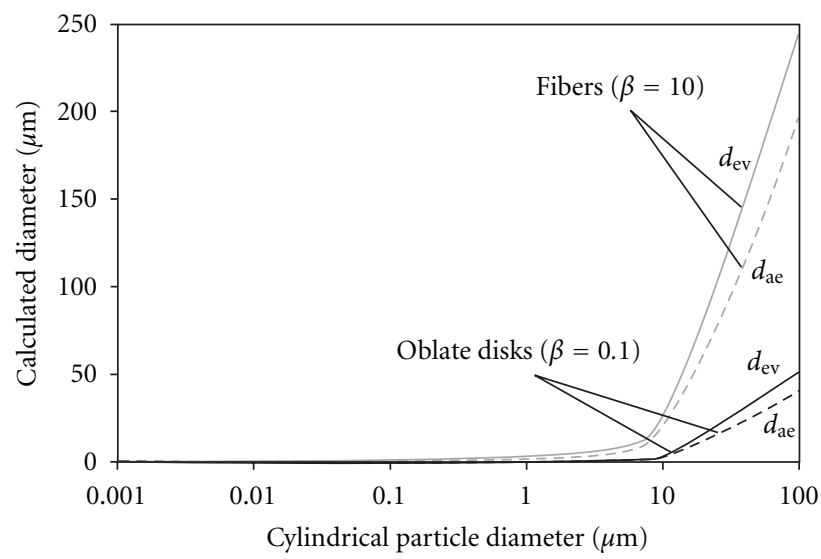

(b)

FIGURE 2: Normalised mobilities $b_{/ /}$and $b_{\perp}$ (a) and calculated diameters $d_{\mathrm{ev}}$ and $d_{\mathrm{ae}}$ (b) of fibers and oblate disks with aspect ratios $\beta$ of 10 and 0.1 , respectively. Diameters $d_{p}$ of the particles range from $0.001 \mu \mathrm{m}$ to $100 \mu \mathrm{m}$.

values for $d_{p}$, while calculated diameters of fibers exhibit a contrary behaviour. This result bears a significant consequence concerning the transport and deposition behaviour of nonspherical particles in the human respiratory tract.

3.2. Particle Deposition in the Human Respiratory Tract. Results of the computations of nonspherical unit-density particle deposition in the human respiratory tract under light-work breathing conditions [23] are summarized in the graphs of Figures 3-5. Concerning total deposition, that is, the particle deposition in the extrathoracic region and in the lungs, significant differences between the investigated particle classes are noticeable. For $d_{p}$ ranging from $0.001 \mu \mathrm{m}$ to $0.5 \mu \mathrm{m}$, fibers exhibit a lower total deposition than comparable spheres, whereby the discrepancy in deposition between the two particle classes positively correlates with the fiber aspect ratio (Figure 3(a)). Within about the same particle diameter range, oblate disks commonly deposit with remarkably higher amounts than related spheres. Contrary to fibers, the difference of total deposition between disks and spheres negatively correlates with the disk aspect ratio, that is, thinner disk deposit more efficiently than thicker 
TABLE 2: Aerodynamic diameters of nonspherical particles with various aspect ratios $\beta$.

\begin{tabular}{|c|c|c|c|c|c|c|}
\hline \multirow{2}{*}{$d_{p}(\mu \mathrm{m})$} & \multicolumn{3}{|c|}{ Fibers } & \multicolumn{3}{|c|}{ Disks, platelets } \\
\hline & $\beta=3$ & $\beta=10$ & $\beta=100$ & $\beta=0.3$ & $\beta=0.1$ & $\beta=0.01$ \\
\hline 0.01 & 0.0151 & 0.0176 & 0.0185 & 0.0070 & 0.0039 & 0.0011 \\
\hline 0.02 & 0.0303 & 0.0354 & 0.0384 & 0.0139 & 0.0079 & 0.0021 \\
\hline 0.05 & 0.0762 & 0.0902 & 0.1053 & 0.0349 & 0.0198 & 0.0053 \\
\hline 0.10 & 0.1532 & 0.1850 & 0.2271 & 0.0700 & 0.0399 & 0.0108 \\
\hline 0.20 & 0.3083 & 0.3786 & 0.4786 & 0.1409 & 0.0808 & 0.0219 \\
\hline 0.50 & 0.7750 & 0.9635 & 1.2425 & 0.3556 & 0.2077 & 0.0571 \\
\hline 1.00 & 1.5536 & 1.9406 & 2.5215 & 0.7144 & 0.4235 & 0.1209 \\
\hline 2.00 & 3.1112 & 3.8964 & 5.0826 & 1.4326 & 0.8569 & 0.2564 \\
\hline 5.00 & 7.7844 & 9.7654 & 12.768 & 3.5882 & 2.1601 & 0.6732 \\
\hline 10.0 & 15.573 & 19.547 & 25.579 & 7.1812 & 4.3338 & 1.3731 \\
\hline
\end{tabular}

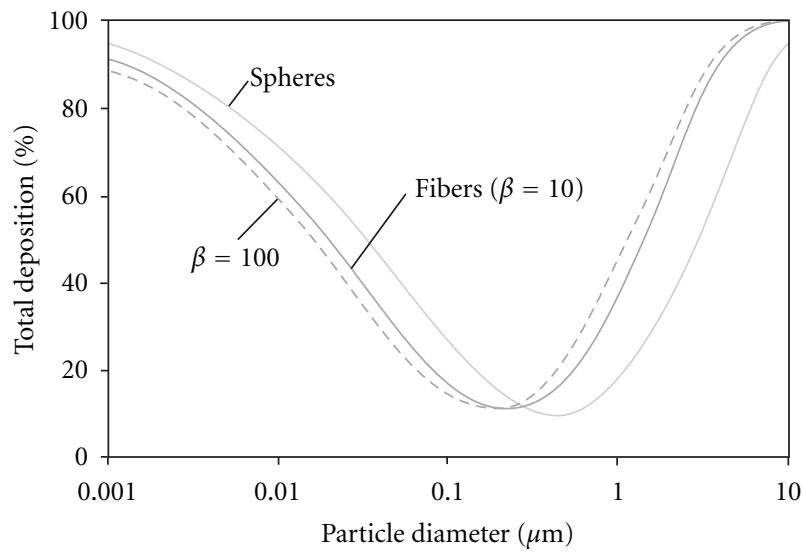

(a)

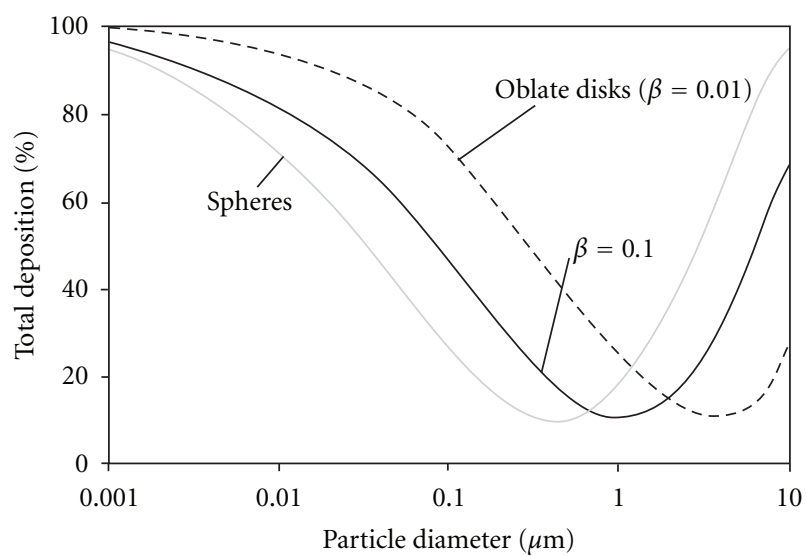

(b)

Figure 3: Total deposition of fibers (a) and platelets (b) with different aspect ratios in the human respiratory tract assuming light work-breathing conditions [23].

ones (Figure 3(b)). For $d_{p}$ ranging from 0.6 to $10 \mu \mathrm{m}$, fibers generally show a higher total deposition than spheres, and the calculated differences are again proportional to the fiber aspect ratio (Figure $3(\mathrm{a})$ ). Oblate disks being attributed to the diameter range noted above deposit to a lower extent than comparable spheres, and the negative correlation between deposition and disk aspect ratio already introduced above is maintained (Figure 3(b)).

Interesting results are also obtained regarding the regional deposition of nonspherical particles, whereby for the computations, three lung regions, namely, the extrathoracic region $(\mathrm{ET})$, the bronchi $(\mathrm{BB})$, and the bronchioli/alveoli $(b b+$ ALV) were distinguished. Simulations were conducted for three different particle sizes to cover a wider range of natural aerosols (Figure 4). For particles with a cylindrical diameter of $0.10 \mu \mathrm{m}$, being the size of, for example, single components from tobacco smoke, bronchiolar and alveolar deposition generally exhibit a remarkable predominance over extrathoracic and bronchial deposition, whereby the effect of particle shape may be evaluated as less significant. Only oblate disks with an aspect ratio of 0.01 slightly deviate from the observed trend insofar as deposition in peripheral lung compartments is reduced by about $15 \%$, and deposition in proximal lung regions is enhanced by the same amount (Figures 4(a) and 4(b)). Concerning 1.0$\mu \mathrm{m}$ particles, the influence of particle shape on regional deposition becomes more significant. While disks are chiefly accumulated in the bronchiolar and alveolar parts of the lungs ( $>80 \%$ of total deposition) again, deposition of fibers is marked by higher variation, because with increasing aspect ratio the extrathoracic and bronchial compartments are targeted to higher amounts by these particles (Figures 4(c) and 4(d)). Deposition of particles with a diameter of $10 \mu \mathrm{m}$ most remarkably depends upon particle shape. While fibers are exclusively deposited in the extrathoracic region, oblate disks of this size class are partly enabled to penetrate into deeper lung regions. Hence, nearly $80 \%$ of disks with an aspect ratio of 0.01 deposit in the peripheral lung compartments, whereas disks with an aspect ratio of 0.1 are accumulated in the extrathoracic and bronchiolar/alveolar region to a comparable extent (about $40 \%$ ) and show their lowest deposition in the bronchi (Figures 4(e) and 4(f)).

The relative particle deposition per airway generation providing some information on local deposition phenomena is illustrated in Figure 5. Spherical and anisometric particles with a cylindrical diameter of $0.10 \mu \mathrm{m}$ are characterised by almost identical deposition patterns with deposition fractions lower than $1 \%$ from the trachea (generation number 1 ) 


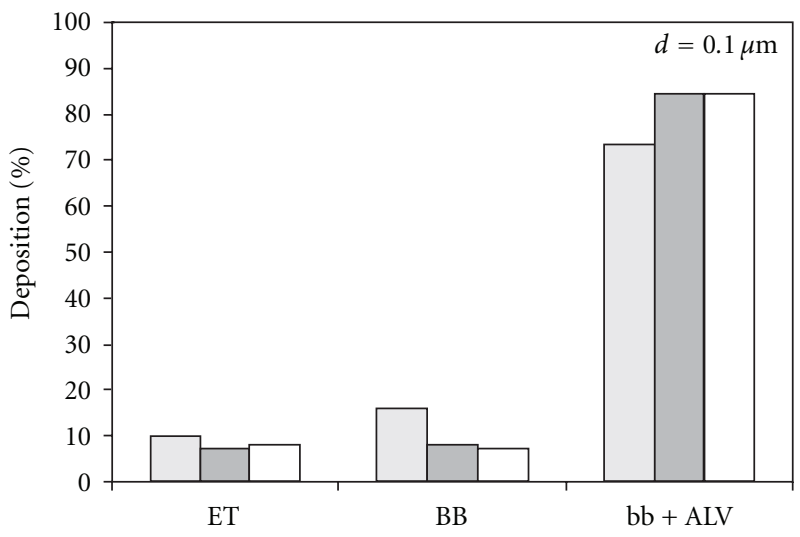

(a)

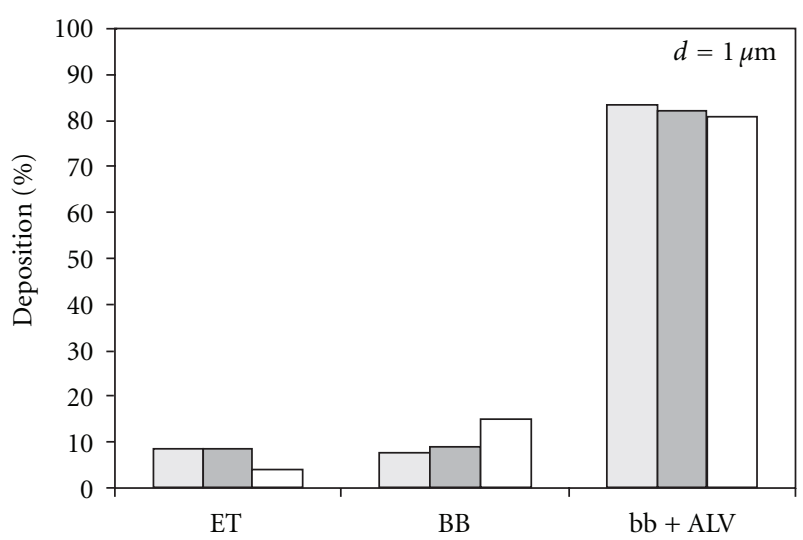

(c)
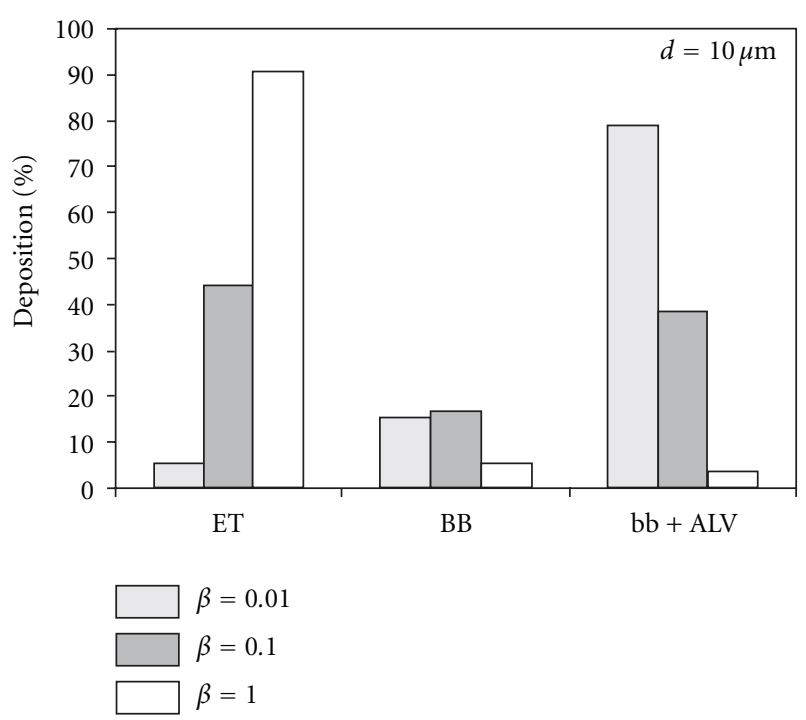

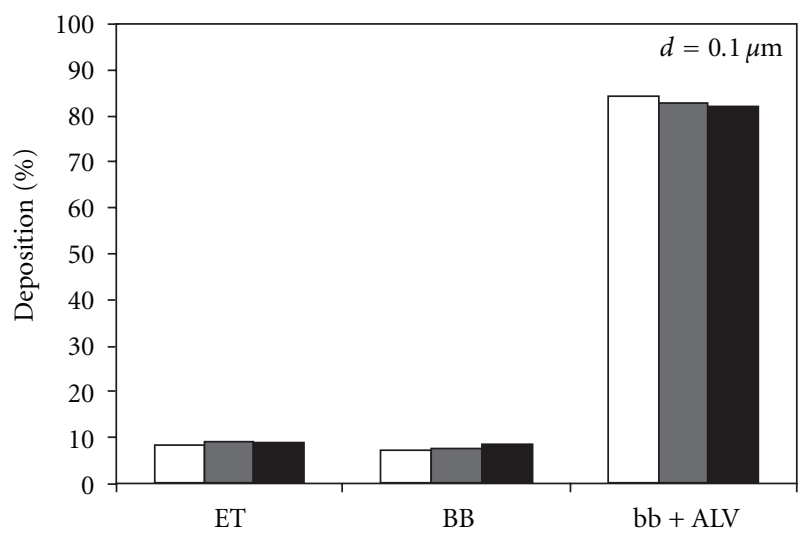

(b)

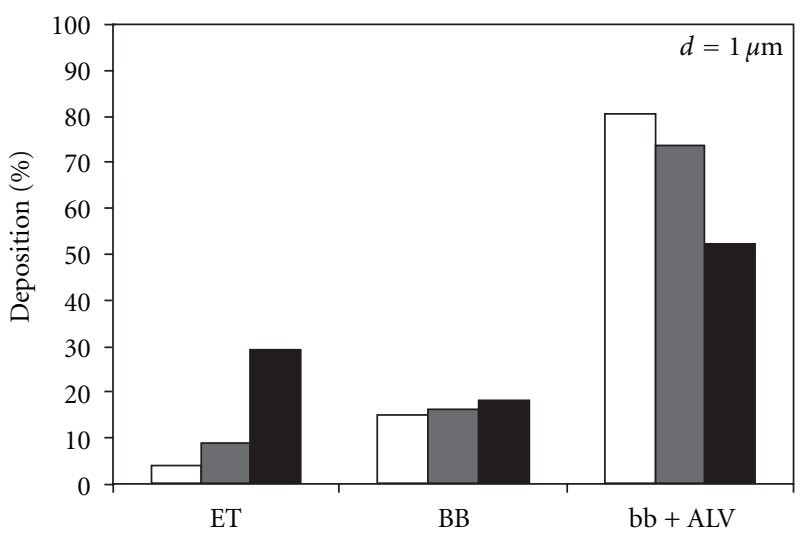

(d)
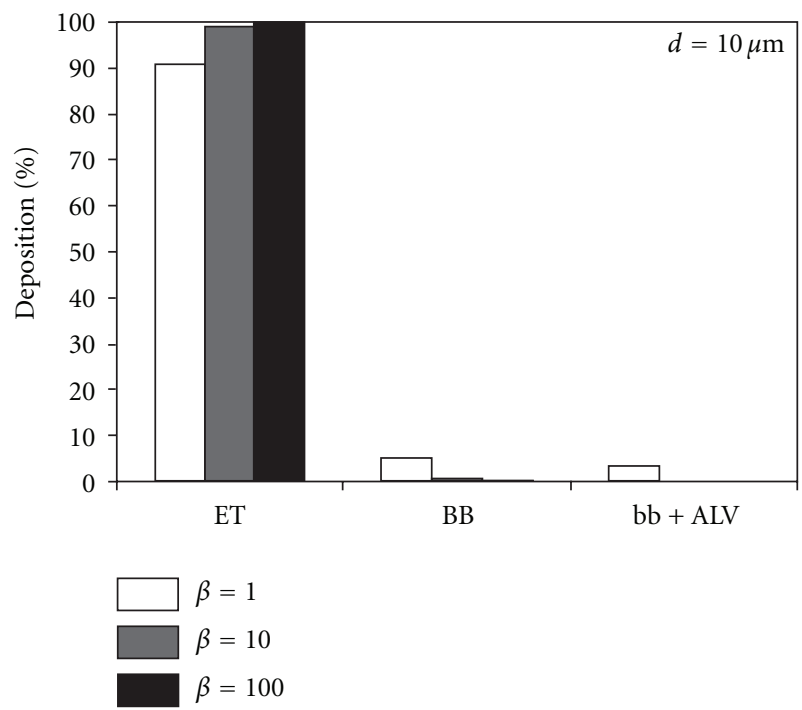

(e)

(f)

FIgURE 4: Regional, that is, extrathoracic (ET), bronchial (BB), and bronchiolar/alveolar (bb + ALV), deposition of spheres $(\beta=1)$, fibers, and oblate disks for three different size categories: (a, b) $0.1 \mu \mathrm{m},(\mathrm{c}, \mathrm{d}) 1.0 \mu \mathrm{m}$, and (e, f) $10 \mu \mathrm{m}$. 


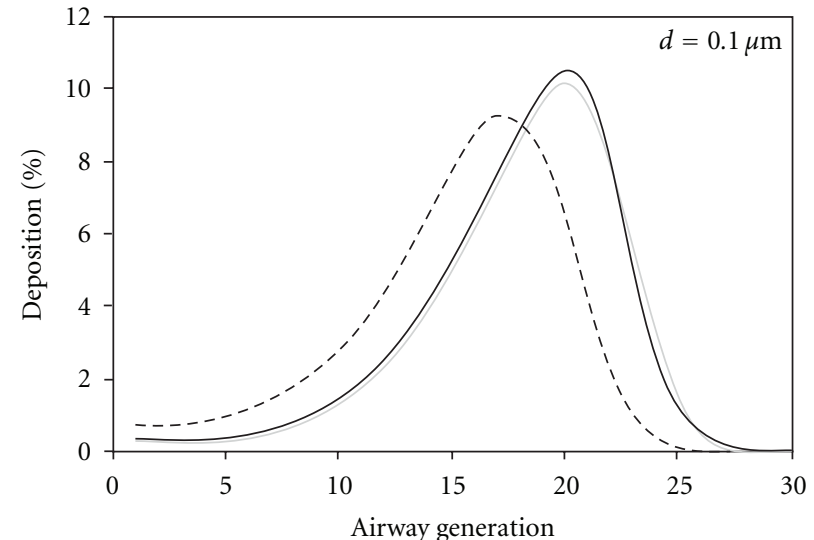

(a)

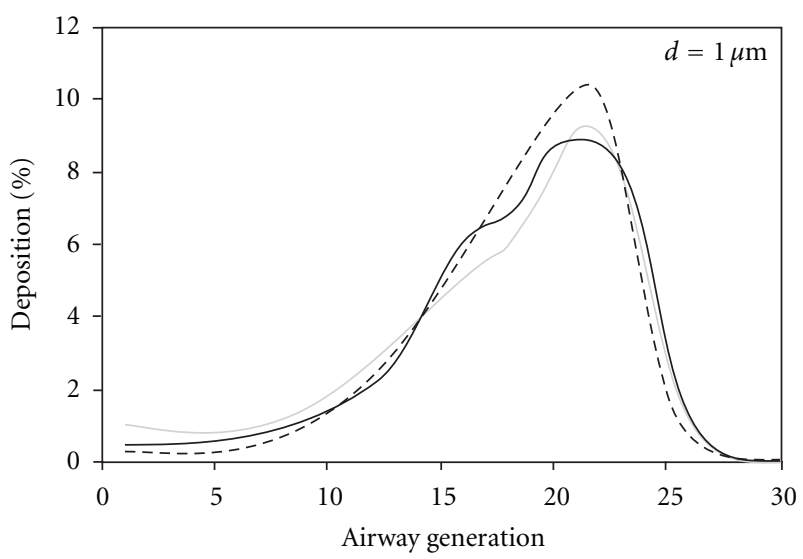

(c)

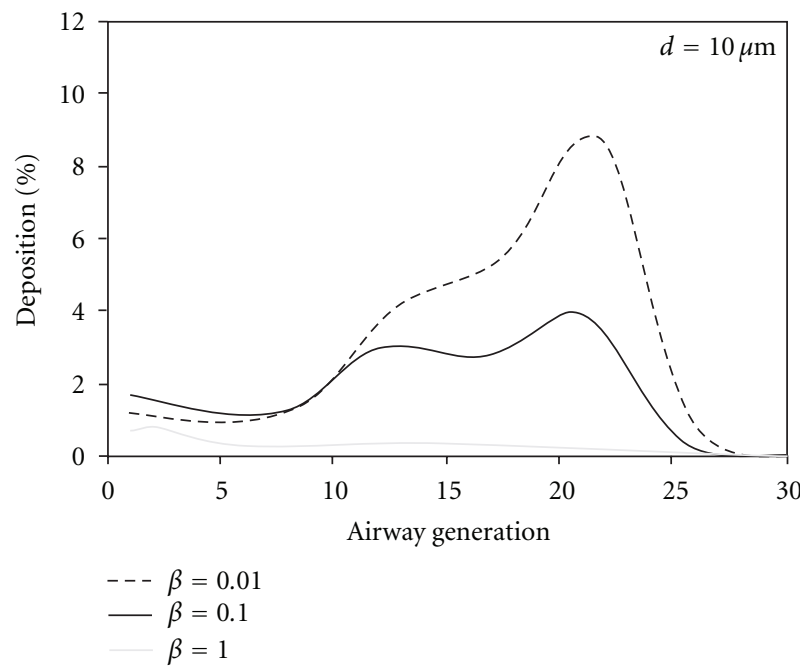

(e)

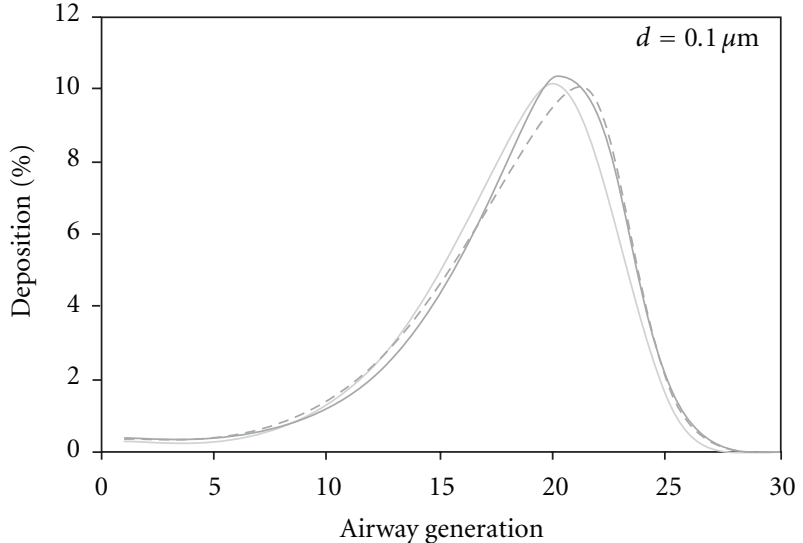

(b)

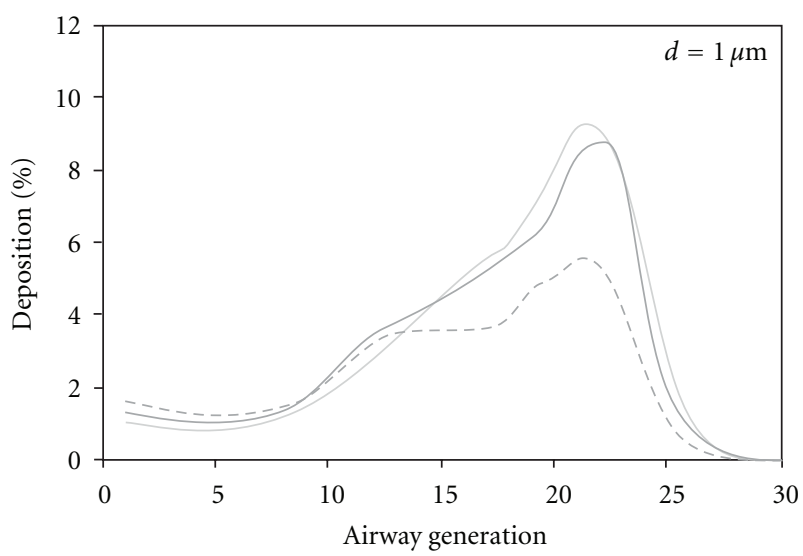

(d)

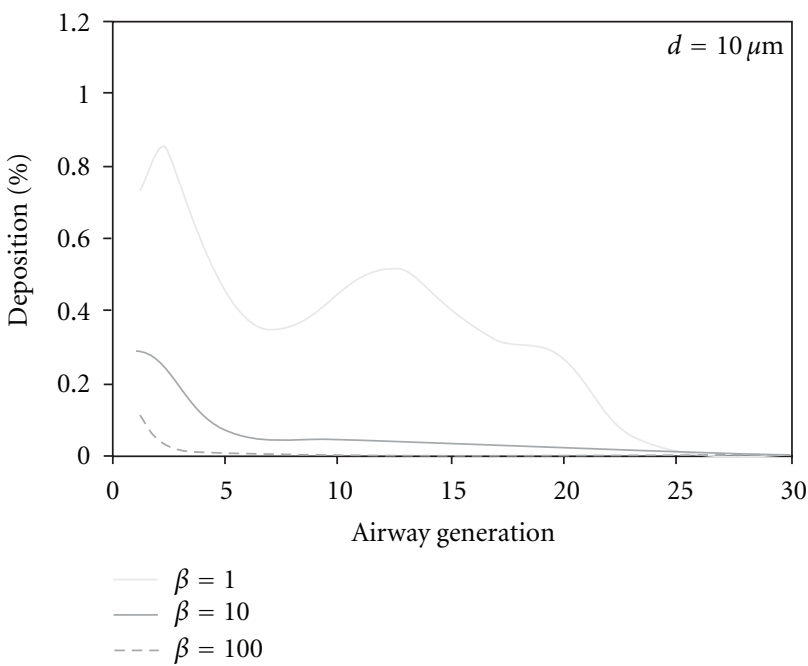

(f)

FIGURE 5: Generation-by-generation deposition of spheres $(\beta=1)$, fibers, and oblate disks belonging to three different size categories: (a, b) $0.1 \mu \mathrm{m},(\mathrm{c}, \mathrm{d}) 1.0 \mu \mathrm{m}$, and (e, f) $10 \mu \mathrm{m}$.

to about airway generation 8 as well as maximum deposition located in airway generation 20 or 21 and ranging from 10 to $11 \%$ (Figures 5(a) and 5(b)). Only very flat disks (aspect ratio 0.01) deviate from this general scheme insofar as deposition is shifted to more proximal airway generations. In this specific case maximum deposition may be located in airway generation 16 and varies between 9 and 10\% (Figure 5(a)). A higher discrepancy of the computed deposition patterns 


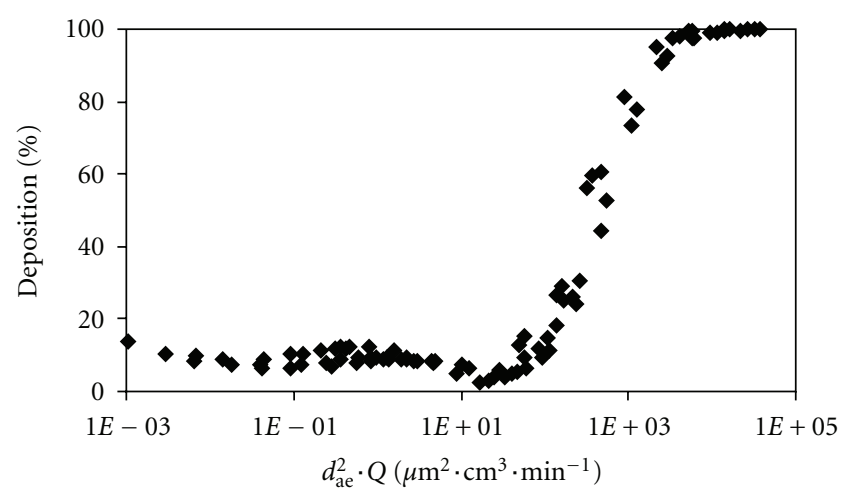

(a)

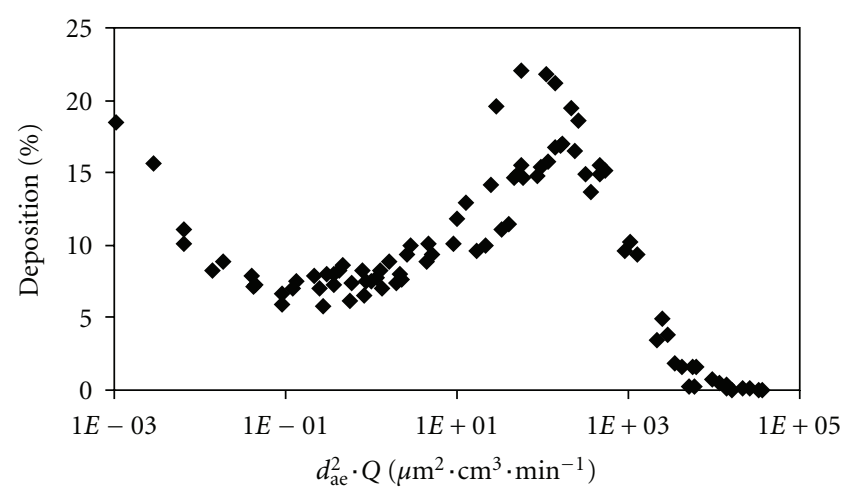

(c)

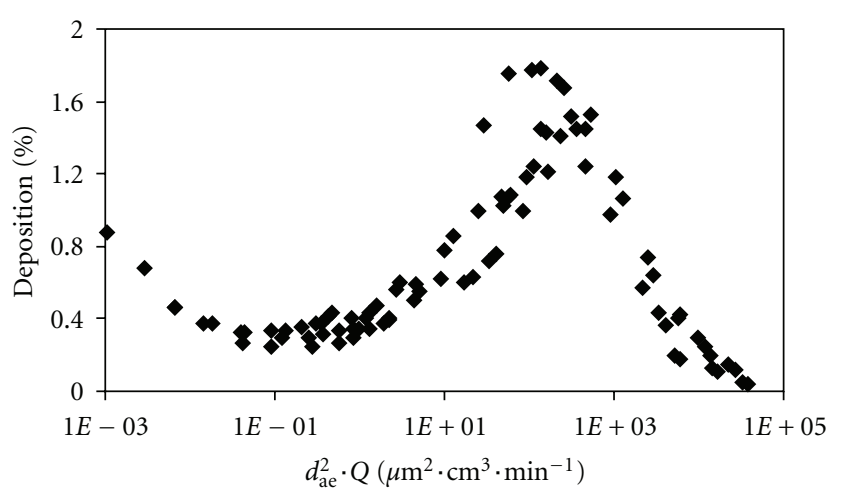

(b)

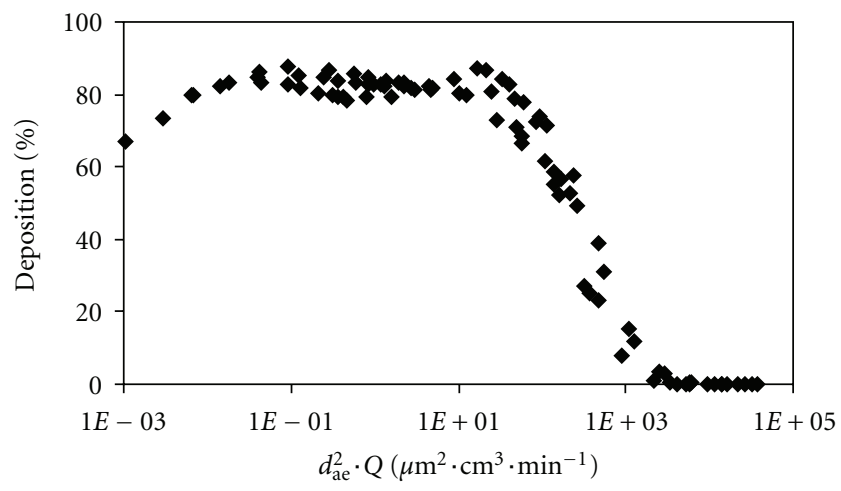

(d)

FIgURE 6: Dependence of extrathoracic (a), tracheal (b), bronchial (c), and bronchiolar/alveolar (d) deposition in the human respiratory tract on $d_{\mathrm{ae}}^{2} \cdot Q$.

may be recognised for particles with a cylindrical diameter of $1.0 \mu \mathrm{m}$. Independent of particle shape, maximum deposition occurs in airway generation 21 or 22, thereby, varying between 5 and $10 \%$ in the case of fibers and between 8 and $10 \%$ in the case of oblate disks. As illustrated in Figure 5(c), the deposition patterns of disks partly exhibit remarkable asymmetries with particle accumulation being significantly enhanced in those airway generations situated proximal to the bifurcation with maximum deposition. This development is even more accentuated for fibers (Figure 5(d)), where the deposition maximum is successively decreased with increasing aspect ratio, tending to a somewhat uniform deposition distribution in intermediate airway generations. Spherical and fibrous particles with a cylindrical diameter of $10 \mu \mathrm{m}$ are chiefly concentrated in the most proximal airway generations, whereby maximum deposition already occurs in the trachea or the directly following airway generation. Overall deposition in the airway bifurcations is dramatically reduced with increasing fiber aspect ratio due to the extrathoracic region serving as a highly effective filter for these elongated particles (Figure 5(f)). A quite different picture is obtained for oblate disks, whose peak deposition may be located in airway generation 20 to 21 again, thereby ranging from 4 to $9 \%$. Contrary to the $0.1-\mu \mathrm{m}$ particles outlined above, this deposition maximum becomes remarkably accentuated with decreasing aspect ratio (Figure 5(e)).
3.3. Deposition of Nonspherical Particles at Different Breathing Conditions. To account for possible health effects due to nonspherical particle deposition in the lungs, regional, that is, extrathoracic, tracheal, bronchial, and bronchiolar/alveolar, deposition of spheres and anisometric particles with various geometric dimensions was computed as a function of the widely used mathematical term $d_{\mathrm{ae}}^{2} \cdot Q$ (Figure 6). In this expression besides particle properties $\left(d_{\mathrm{ae}}\right)$ also essential characteristics of the air flow $(Q)$ are included. For the investigation presented here, $Q$ ranged from $10^{4} \mathrm{~cm}^{3} \cdot \mathrm{min}^{-1}$ (breathing conditions during sleep [23]) to $12 \cdot 10^{5} \mathrm{~cm}^{3} \cdot \mathrm{min}^{-1}$ (breathing condition during heavy work [23]). In the extrathoracic region particle deposition takes values $<20 \%$ for $d_{\mathrm{ae}}^{2} \cdot Q$ ranging from $10^{-3}$ to $10^{1} \mu \mathrm{m}^{2} \cdot \mathrm{cm}^{3} \cdot \mathrm{min}^{-1}$ but becomes highly significant for $d_{\mathrm{ae}}^{2} \cdot Q>10^{2} \mu \mathrm{m}^{2} \cdot \mathrm{cm}^{3} \cdot \mathrm{min}^{-1}$. In the case of $d_{\mathrm{ae}}^{2} \cdot Q$ exceeding $10^{3} \mu \mathrm{m}^{2} \cdot \mathrm{cm}^{3} \cdot \mathrm{min}^{-1}$ due to, for example, an increase of the breathing frequency or an enhancement of the aerodynamic particle diameter deposition continuously approaches $100 \%$ (Figure 6(a)). In the trachea highest particle deposition (ca. 1.8\%) may be recognised for $d_{\mathrm{ae}}^{2} \cdot Q$ amounting to about $10^{2} \mu \mathrm{m}^{2} \cdot \mathrm{cm}^{3} \cdot \mathrm{min}^{-1}$. Another remarkably smaller deposition peak is located at very low values for $d_{\mathrm{ae}}^{2} \cdot \mathrm{Q}\left(10^{-3} \mu \mathrm{m}^{2} \cdot \mathrm{cm}^{3} \cdot \mathrm{min}^{-1}\right)$, underlining the significance of Brownian diffusion as a main deposition mechanism in the tracheal airway tube (Figure 6(b)). Regarding 
the bronchial lung compartment deposition of particles and its dependence upon $d_{\mathrm{ae}}^{2} \cdot Q$ exhibits some correspondences with the trachea insofar as respective deposition peaks are also located at $d_{\mathrm{ae}}^{2} \cdot Q=10^{2} \mu \mathrm{m}^{2} \cdot \mathrm{cm}^{3} \cdot \mathrm{min}^{-1}$ and $d_{\mathrm{ae}}^{2} \cdot Q=10^{-3} \mu \mathrm{m}^{2} \cdot \mathrm{cm}^{3} \cdot \mathrm{min}^{-1}$. Differences between the two parts of the respiratory tract chiefly concern the amount of deposited material which in the bronchi is about 10 times higher than in the trachea (Figure 6(c)). In the bronchiolar/alveolar lung compartment highest particle deposition $(>80 \%)$ may be expected for $d_{\mathrm{ae}}^{2} \cdot Q$ ranging from $10^{-2}$ to $10^{1} \mu \mathrm{m}^{2} \cdot \mathrm{cm}^{3} \cdot \mathrm{min}^{-1}$. While for lower values of $d_{\mathrm{ae}}^{2} \cdot Q$ particle deposition is subject to a slight decrease, higher values cause a sharp drop of deposition, so that, for $d_{\mathrm{ae}}^{2} \cdot Q>$ $5 \cdot 10^{3} \mu \mathrm{m}^{2} \cdot \mathrm{cm}^{3} \cdot \mathrm{min}^{-1}$, it may be evaluated as negligible (Figure 6(d)).

\section{Discussion}

Since environmental aerosols mainly contain particles that deviate from ideal spherical shape, detailed knowledge concerning the deposition of such nonspherical particulate matter in the human lungs represents an important basis in modern pulmology. In the past, theoretical description of the transport and deposition of anisometric particles in the human respiratory tract has chiefly been conducted by using the aerodynamic diameter concept $[1,9-11,13,14,19,24]$ and, in rarer cases, with the help of numerical approaches $[16,17,25]$. Meanwhile the former theoretical concept has been subject to numerous improvements and refinements, so that it has been implemented in the stochastic particle transport and deposition code introduced by Koblinger and Hofmann [18], thereby providing reliable and highly plausible results. Core of the mathematic formula expressing the aerodynamic diameter of fibers and oblate disks is the so-called dynamic shape factor $\chi$ that includes the deviation of the particle shape from a sphere [12, 19, 24, 26-28]. In the study presented here $\chi$ commonly takes values greater than 1 for both fibrous particles and oblate disks. This is in good correspondence with experimental and theoretical data provided by the pioneering works of the 1970s and 1980s [12, 19, 24] (note: the dynamic shape factor $\chi$ presented in this work corresponds with the volume shape factor of Davies.). As an essential consequence of the calculated $\chi$-values fibrous and disk-shaped particles yielding an identical equivalent volume diameter, $d_{\mathrm{ev}}$ are marked by different aerodynamic diameters $d_{\mathrm{ae}}$ and, hence, by very individual deposition patterns in the human lungs (Table 2, Figures 3-5).

Regarding total particle deposition in the human respiratory tract, fibers and oblate disks exhibit partly significant characteristics (Figure 3). Within the particle size range from 0.001 to about $0.5 \mu \mathrm{m}$, including the important class of ultrafine particles, disks commonly show higher total deposition than fibers, whereas for particle sizes ranging from $>0.5 \mu \mathrm{m}$ to $10 \mu \mathrm{m}$ the inverse behaviour is observed. These phenomena are mainly founded upon the circumstance that equivalent volume diameters of fibers and disks with the same cylindrical diameter may differ by a remarkable order of magnitude; that is, disks show a smaller $d_{\mathrm{ev}}$ than fibers. For the submicrometer size range, this causes an increased

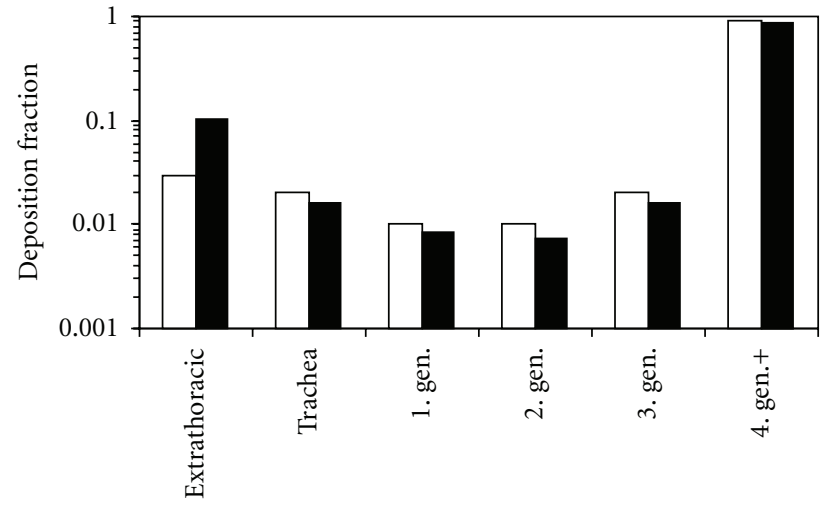

(a)

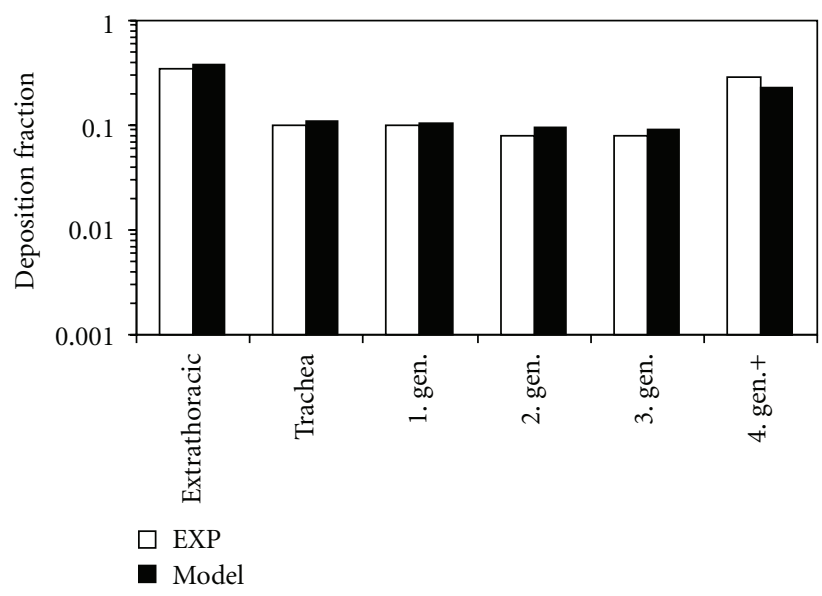

(b)

Figure 7: Comparison between experimental [1] and theoretical particle deposition data for model validation: (a) inhalative flow: $15 \mathrm{~L} \mathrm{~min}^{-1}$, fiber length: $10-20 \mu \mathrm{m}$; (b) inhalative flow: $45 \mathrm{~L} \mathrm{~min}^{-1}$, fiber length: $60-70 \mu \mathrm{m}$. It has to be additionally noted that the experimental performance was carried out on the basis of a silicon rubber cast of the upper human airways. Therefore, the geometry of this replica had to be exactly considered in the theoretical model before the conduction of any validation calculations.

deposition probability of disks by Brownian diffusion [18]. The predominance of fiber deposition in the micrometer size range is also based on the different $d_{\mathrm{ev}}$-values, whereby in this specific case fibers are more effectively deposited due to an enhanced effect of inertial impaction and interception [18]. The particle-shape-induced phenomena standing behind total deposition in the respiratory tract are also responsible for regional and airway generation-by-generation deposition patterns. Concerning the behaviour of fibers, theoretical values presented here show a good agreement with data obtained from experiments with a replica of the upper human lung airways [1]. As illustrated in Figure 7, differences between the experimental data sets and theoretical predictions are on the order of 2 to 20 percent. A serious exception is given for extrathoracic deposition (differences up to 
70 percent, Figure $7(a)$ ), exhibiting a significant weakness of the theoretical approach. It has to be added, however, that deposition of spherical particles with comparable diameter undercuts fiber deposition by about one third, strictly underlining the significance of geometric particle properties for theoretical calculations. Relative errors of the experimental results and theoretical computations are on the order of $10 \%$.

Unfortunately, any comparison of the results generated in this study with calculations derived from numerical simulation methods using either the Lagrangian-Eulerian technique or DNS is not possible because numerical models are not able to define appropriate anisometric particles hitherto. In future models the effect of fibrosis generation due to enhanced alveolar deposition of dust particles and fibers will be considered more in detail.

\section{Conclusions}

Summing up the results of fiber and disk deposition in different lung regions and airway generations, preferred deposition sites of anisometric particles are the airway tubes and, depending upon particle size, also the alveoli. From these findings, it can be concluded that these particle classes may represent tremendous occupational hazards, especially if they are attached with radioactive elements or heavy metals $[1,23]$. As could be theoretically shown for specific particle sizes, tubular and alveolar deposition can be reduced with increasing flow rate (Figure 6) [18, 23], which plays an important role in the debate regarding the inhalation of hazardous aerosols by light and heavy workers. Although numerous problems of anisometric particle deposition can be clarified with the current approaches and the model presented here, further scientific efforts are necessary in the future to successively substitute the ideal particle shapes such as spheres, fibers, and platelets by more realistic shapes.

\section{References}

[1] W. C. Su and Y. S. Cheng, "Deposition of fiber in a human airway replica," Journal of Aerosol Science, vol. 37, no. 11, pp. 1429-1441, 2006.

[2] M. Lippmann, "Effects of fiber characteristics on lung deposition, retention, and disease," Environmental Health Perspectives, vol. 88, pp. 311-317, 1990.

[3] T. Myojo, "Deposition of fibrous aerosol in model bifurcating tubes," Journal of Aerosol Science, vol. 18, no. 3, pp. 337-347, 1987.

[4] Y. K. Chen and C. P. Yu, "Sedimentation of fibers from laminar flows in a horizontal circular duct," Aerosol Science and Technology, vol. 14, no. 3, pp. 343-347, 1991.

[5] F. S. Cai and C. P. Yu, "Inertial and interceptional deposition of spherical particles and fibers in a bifurcating airway," Journal of Aerosol Science, vol. 19, no. 6, pp. 679-688, 1988.

[6] T. W. Hesterberg and G. A. Hart, "Synthetic vitreous fibers: a review of toxicology research and its impact on hazard classification," Critical Reviews in Toxicology, vol. 31, no. 1, pp. 1-53, 2001.

[7] O. Kamstrup, A. Ellehauge, C. G. Collier, and J. M. G. Davis, "Carcinogenicity studies after intraperitoneal injection of two types of stone wool fibres in rats," Annals of Occupational Hygiene, vol. 46, no. 2, pp. 135-142, 2002.

[8] International Agency for Research on Cancer (IARC), IARC Monographs on the Evaluation of Carcinogenic Risks to Humans, Vol 81: Man-Made Vitreous Fibers, IARC Press, Lyon, France, 2002.

[9] J. G. Slowik, K. Stainken, P. Davidovits et al., "Particle morphology and density characterization by combined mobility and aerodynamic diameter measurements. Part 2: application to combustion-generated soot aerosols as a function of fuel equivalence ratio," Aerosol Science and Technology, vol. 38, no. 12 , pp. 1206-1222, 2004.

[10] P. F. DeCarlo, J. G. Slowik, D. R. Worsnop, P. Davidovits, and J. L. Jimenez, "Particle morphology and density characterization by combined mobility and aerodynamic diameter measurements. Part 1: theory," Aerosol Science and Technology, vol. 38, no. 12, pp. 1185-1205, 2004.

[11] A. Virtanen, J. Ristimäki, and J. Keskinen, "Method for measuring effective density and fractal dimension of aerosol agglomerates," Aerosol Science and Technology, vol. 38, no. 5, pp. 437-446, 2004.

[12] W. Stöber, "Dynamic shape factors of nonspherical aerosol particles," in Assessment of Airborne Particles, T. Mercer, Ed., pp. 247-289, Charles C. Thomas Publisher, Springfield, Ill, USA, 1972.

[13] Y. T. Dai and C. P. Yu, "Alveolar deposition of fibers in rodents and humans," Journal of Aerosol Medicine, vol. 11, no. 4, pp. 247-258, 1998.

[14] Z. L. Arsenijevic, Z. B. Grbavcic, R. V. Garic-Grulovic, and F. K. Zdanski, "Determination of non-spherical particle terminal velocity using particulate expansion data," Powder Technology, vol. 103, no. 3, pp. 265-273, 1999.

[15] R. Sturm and W. Hofmann, "A computer program for the simulation of fiber deposition in the human respiratory tract," Computers in Biology and Medicine, vol. 36, no. 11, pp. 12521267, 2006.

[16] B. Asgharian and S. Anijilvel, "Movement and deposition of fibers in an airway with steady viscous flow," Aerosol Science and Technology, vol. 22, no. 3, pp. 261-270, 1995.

[17] M. Shams, G. Ahmadi, and H. Rahimzadeh, "Transport and deposition of flexible fibers in turbulent duct flows," Journal of Aerosol Science, vol. 32, no. 4, pp. 525-547, 2001.

[18] L. Koblinger and W. Hofmann, "Monte Carlo modeling of aerosol deposition in human lungs. Part I: simulation of particle transport in a stochastic lung structure," Journal of Aerosol Science, vol. 21, no. 5, pp. 661-674, 1990.

[19] G. Kasper, "Dynamics and measurement of smokes. I. Size characterization of nonspherical particles," Aerosol Science and Technology, vol. 1, no. C, pp. 187-199, 1982.

[20] L. Koblinger, "Analysis of human lung morphometric data for stochastic aerosol deposition calculations," Physics in Medicine and Biology, vol. 30, no. 6, article 004, pp. 541-556, 1985.

[21] B. Haefeli-Bleuer and E. R. Weibel, "Morphometry of the human pulmonary acinus," Anatomical Record, vol. 220, no. 4, pp. 401-414, 1988.

[22] H. C. Yeh and G. M. Schum, "Models of human lung airways and their application to inhaled particle deposition," Bulletin of Mathematical Biology, vol. 42, no. 3, pp. 461-480, 1980.

[23] International Commission on Radiological Protection (ICRP), Human Respiratory Tract Model for Radiological Protection, Publication 66, Pergamon Press, Oxford, UK, 1994.

[24] C. N. Davies, "Particle-fluid interaction," Journal of Aerosol Science, vol. 10, no. 5, pp. 477-513, 1979. 
[25] I. Balásházy, T. B. Martonen, and W. Hofmann, "Fiber deposition in airway bifurcations," Journal of Aerosol Medicine, vol. 3, pp. 243-260, 1990.

[26] R. Sturm and W. Hofmann, "A theoretical approach to the deposition and clearance of fibers with variable size in the human respiratory tract," Journal of Hazardous Materials, vol. 170, no. 1, pp. 210-218, 2009.

[27] A. Hölzer and M. Sommerfeld, "Lattice Boltzmann simulations to determine drag, lift and torque acting on nonspherical particles," Computers and Fluids, vol. 38, no. 3, pp. 572-589, 2009.

[28] M. Sommerfeld, "Particle dispersion in turbulent flow. The effect of particle size distribution," Particle \& Particle Systems Characterization, vol. 7, no. 4, pp. 209-220, 1990. 

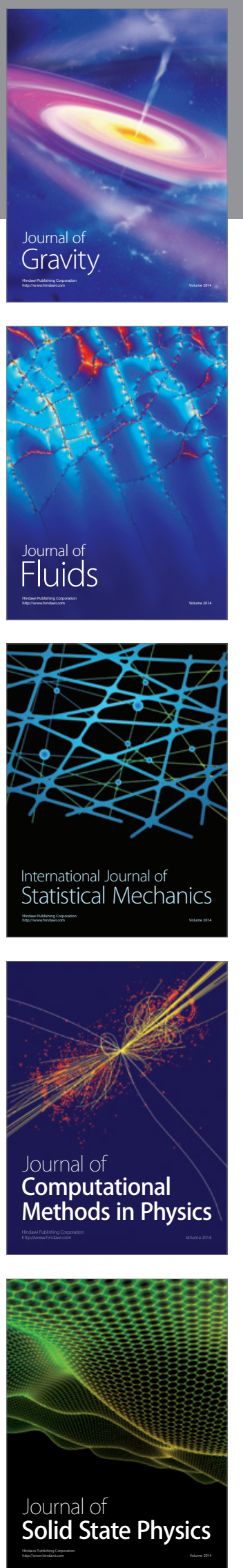

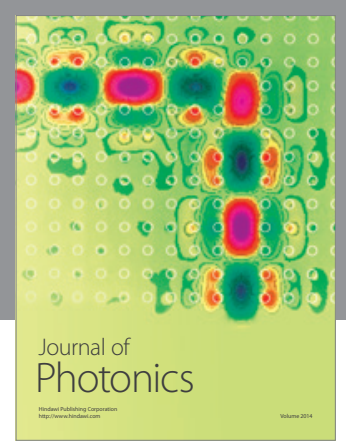

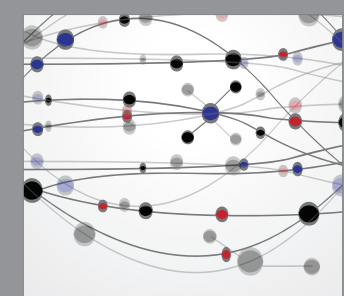

The Scientific World Journal
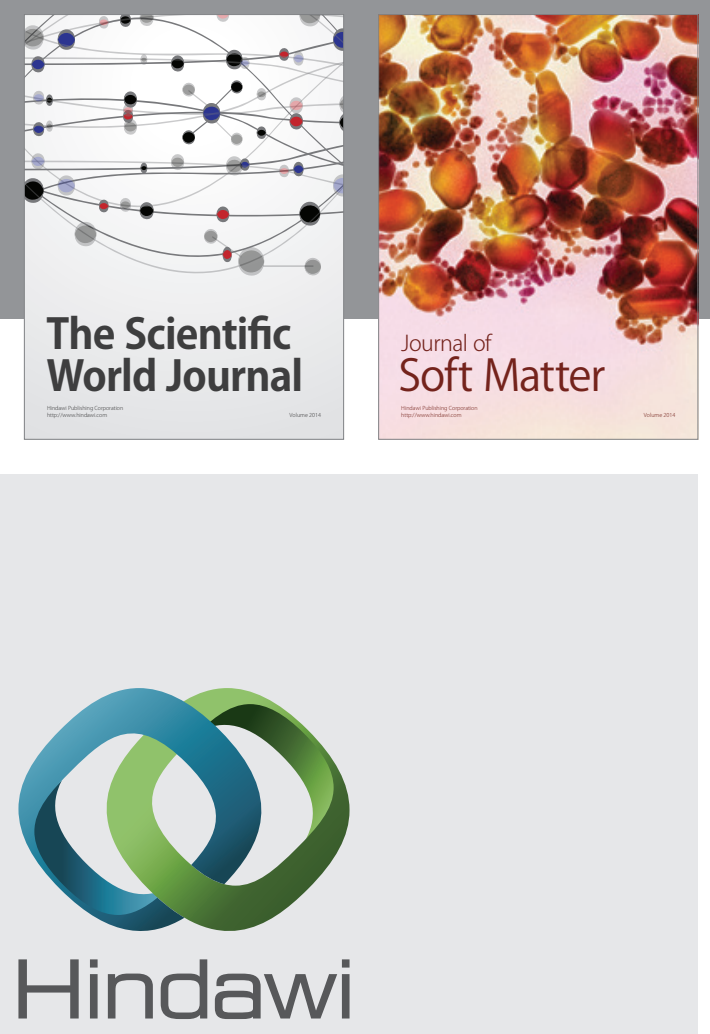

Submit your manuscripts at

http://www.hindawi.com
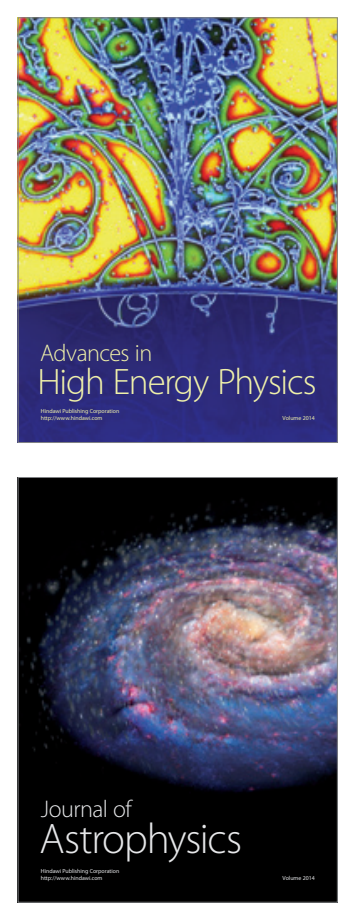
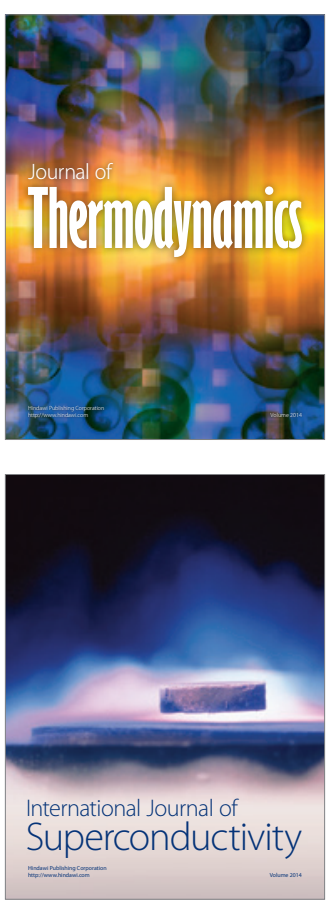
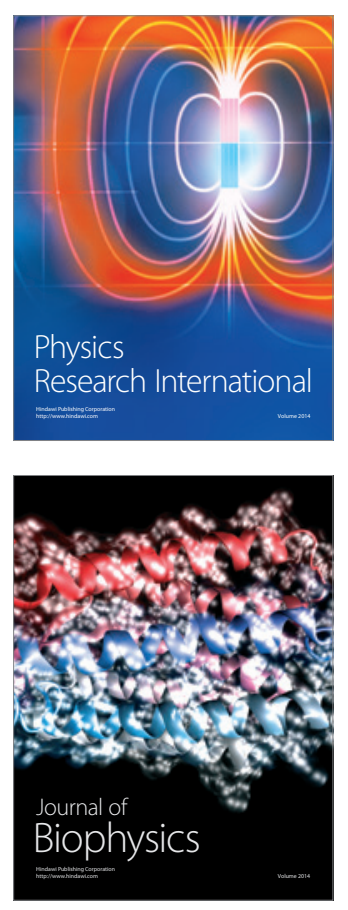
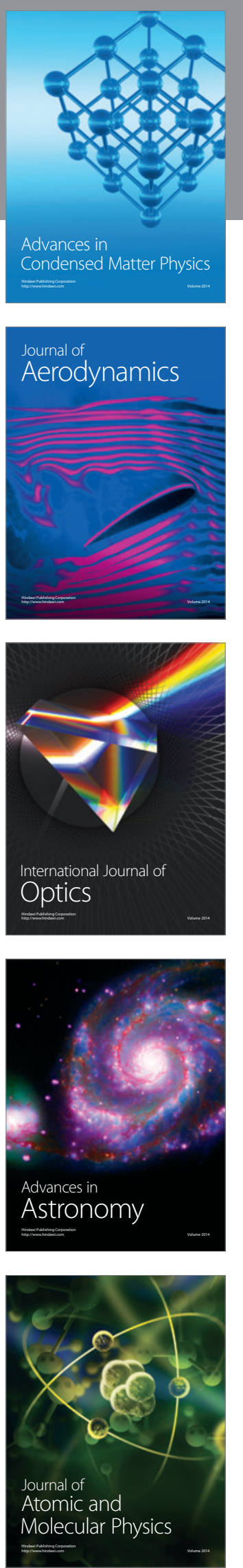